\title{
ASYMPTOTIC PROPERTIES OF GAUSSIAN RANDOM FIELDS
}

\author{
BY
}

\section{CLIFFORD QUALLS AND HISAO WATANABE}

ABSTRACT. In this paper we study continuous mean zero Gaus sian random fields $X(p)$ with an $N$-dimensional parameter and having a correlation function $\rho(p, q)$ for which $1-\rho(p, q)$ is asymptotic to a regularly varying (at zero) function of the distance dis $(p, q)$ with exponent $0<\alpha \leq 2$. For such random fields, we obtain the asymptotic tail distribution of the maximum of $X(p)$ and an asymptotic almost sure property for $X(p)$ as $|p| \rightarrow \infty$. Both results generalize ones previously given by the authors for $N=1$.

1. Introduction. In this paper, we are concerned with a real continuous Gaussian process with an $N$-dimensional parameter space. We denote such a random field by $X=\left\{X(p) ; p \in R^{N}\right\}$, and we assume without loss of generality that each $X(p)$ has mean zero and variance one. We also assume throughout this paper that $X$ satisfies, for some positive constants $C_{1}$ and $C_{2}$,

(A) $\quad E\left((X(p)-X(q))^{2}\right) \leq 2 C_{2}|p-q|^{a} H(|p-q|)$ and

(B) $\quad E\left((X(p)-X(q))^{2}\right) \geq 2 C_{1}|p-q|^{\alpha} H(|p-q|)$

for all $p, q$ such that $|p-q| \leq$ some $\delta$, where $0<\alpha \leq 2$ and $H(s)$ is a slowly varying function (at zero). Here and throughout the paper, we define $|p|=$ $\left(\Sigma_{i=1}^{N} p_{i}^{2}\right)^{1 / 2}$, where $p=\left(p_{1}, \cdots, p_{N}\right)$. For the preliminary proofs of $\$ 2$, we further restrict $X$ to be a stationary isotropic Gaussian random field satisfying

$$
\rho(p, q)=1-|p-q|^{\alpha} H(|p-q|)+o\left(|p-q|^{a} H(|p-q|)\right) \text { as }|p-q| \rightarrow 0,
$$

where $\rho(p, q)=E(X(p) X(q))$ is the correlation function of $X$.

In $\$ 2$, we give the asymptotic tail distribution of the maximum $Z(D)=$ $\max _{p \in D} X(p)$, where $D$ is an open bounded set with Lebesgue measure $\mu(D)=$ $\mu(\bar{D})$. This is done first in Theorem 2.1 for stationary isotropic Gaussian random fields satisfying condition (1.2), then extended in the Corollary to Theorem 2.1 to obtain asymptotic bounds of the tail distribution of $Z(D)$ for Gaussian random fields satisfying condition (1.1) for all $p, q \in D$ with $|p-q|<\delta$. Lemma 2.3 and

Received by the editors January 20, 1972.

AMS (MOS) subject classifications (1970). Primary 60F20, 60G15, 60G17.

Key words and phrases. Regular variation, slow variation, random fields, supremum of stochastic processes, isotropic, stationary, 0-1 law. 
its corollary are useful discrete versions of Theorem 2.1 and its corollary. The results of this $\$ 2$ generalize Theorem 2.1 in Qualls-Watanabe [4] and the results of Pickands [2] from continuous time Gaussian processes to Gaussian random fields.

In $\$ 3$. we study the event

$$
A_{\phi}=\left\{\exists r_{0} ; X(p) \leq \phi(|p|) \text { for all } p \text { satisfying }|p| \geq r_{0}\right\}
$$

for arbitrary nondecreasing functions $\phi$. In more descriptive language, the complement of $A_{\phi}$ occurs if $X(p)$ crosses the surface $\phi(|p|)$ infinitely often as $|p| \rightarrow \infty$. For a fairly wide class of Gaussian processes, we can expect that $P\left(A_{\phi}\right)=0$ or 1 . By using the results of $\$ 2$, we give a criterion in terms of $\phi$ for deciding whether the probability of the event $A_{\phi}$ is 0 or 1 . In order to obtain $P\left(A_{\phi}\right)=0$, the only hypothesis necessary (beside the requirement on $\phi$ ) is that part (A) of condition (1.1) holds for all $p, q$ such that $|p|,|q| \geq$ some $T_{1}$ and $|p-q|<$ some $\delta_{1}$. To obtain $P\left(A_{\phi}\right)=1$, we require part (B) of condition (1.1) to hold for all $p, q$ such that $|p|,|q| \geq$ some $T_{2}$ and $|p-q|<$ some $\delta_{2}$, and we need a mixing condition

(C) $\rho(p, p+q)=O\left(|q|^{-\gamma}\right)$ uniformly in $p$ as $|q| \rightarrow \infty$,

for some $y>0$. This is an extension of the results in Qualls-Watanabe [4] to Gaussian random fields.

The proofs of these results of course bear similarities to those for the case $N=1$. However, we take a somewhat different point of view in the present paper; and the details are considerably different for $N>1$. A good source of general information about Gaussian processes and random fields is the book by Cramér and Leadbetter, Stationary and related stochastic processes, Wiley, New York, 1967.

2. The asymptotic distribution of the maximum. We first list some definitions and properties of regular varying functions that will be required in the following. One general reference on regular variation is Feller [1].

Definition 2.1. A positive function $H(x)$ defined for $x>0$ varies slowly at zero if for all $t>0$

$$
\lim _{x \rightarrow 0} \frac{H(t x)}{H(x)}=1 .
$$

Definition 2.2. A positive function $Q(x)$ defined for $x>0$ varies regularly at zero with exponent $a \geq 0$ if for all $t>0$

$$
\lim _{x \rightarrow 0} \frac{Q(t x)}{Q(x)}=t^{\alpha}
$$

A function $Q(x)$ satisfies (2.2) if and only if $Q(x)=x^{\alpha} H(x)$, where $H(x)$ varies slowly. Let $Q(x)$ vary regularly with exponent $\alpha \geq 0$ and $H(x)$ vary 
slowly at zero. Then the following properties hold.

(2.3) The limits (2.1) and (2.2) converge uniformly in $t$ on any compact subset of the half line $(0, \infty)$.

(2.4) For any $\epsilon>0$, we have

$$
\lim _{x \rightarrow 0} x^{-\epsilon} H(x)=\infty \text { and } \lim _{x \rightarrow 0} x^{\epsilon} H(x)=0 .
$$

(2.5) If $H(x)$ is a slowly varying function at zero, then for any $\epsilon>0$ and $t_{0}>1$, there exists a $\delta>0$ such that $t^{-\epsilon} \leq H(t x) / H(x) \leq t^{\epsilon}$ for all $x>0$ and all $t \geq t_{0}$ satisfying $t x<\delta$.

(2.6) Also for any $\epsilon>0$ and positive $t_{0}<1$, there exists a $\delta>0$ such that $t^{\epsilon} \leq H(t x) / H(x) \leq t^{-\epsilon}$ for all positive $t \leq t_{0}$ and all $x>0$ satisfying $x<\delta$.

A Gaussian random field $X$ will be called stationary or homogeneous if for each choice of points $p_{1}, p_{2}, \cdots, p_{k}$ in $R^{N}$ the joint distribution of $X\left(p_{1}+q\right)$, $\cdots, X\left(p_{k}+q\right)$ does not depend on $q$ in $R^{N}$. Also $X$ will be called isotropic if the correlation function $\rho(p, q)=E(X(p) X(q))$ depends only on the distance $|p-q|$.

Define the function $\sigma^{2}$ by $\sigma^{2}(p, q)=E\left(|X(p)-X(q)|^{2}\right)$ and also let $\tilde{\sigma}^{2}(s)=2|s|^{\alpha} H(s)$, where $H($.$) is a slowly varying function at zero and 0<\alpha \leq 2$. When $X$ is isotropic, $\sigma^{2}(p, q)$ can be written as $\sigma^{2}(|p-q|)$. We assume without loss of generality that $\widetilde{\sigma}($.$) is monotone near the origin. Define$

$$
\begin{aligned}
& A_{1}(\tau)=\inf \{\sigma(p, q) / \tilde{\sigma}(|p-q|) ; 0<|p-q| \leq \tau\}, \\
& A_{2}(\tau)=\sup \{\sigma(p, q) / \tilde{\sigma}(|p-q|) ; 0<|p-q| \leq \tau\} .
\end{aligned}
$$

For a space parameter of dimension $N>1$, and $X$ satisfying the condition (1.2), there is no loss in generality in taking $0<A_{1}(\tau) \leq A_{2}(\tau)<\infty$ for all $\tau>0$. For $N=1$, there is very little loss in generality in assuming $A_{1}(\tau)>0$ for all $\tau$ of interest; the excluded periodic case is discussed in [3].

Theorem 2.1. Let $X$ be a stationary and isotropic, Gaussian random field satisfying condition (1.2). Let $D$ be a bounded open set in $R^{N}$ for whicb the Lebesgie measure $\mu(D)=\mu(\bar{D})$. Then for $Z(D)=\sup _{p \in D} X(p)$ we have

$$
\lim _{x \rightarrow \infty} \frac{P(Z(D)>x)}{\mu(D) \psi(x)\left(\tilde{\sigma}^{-1}(1 / x)\right)^{-N}}=H_{a}
$$

$$
\equiv \lim _{T \rightarrow \infty} T^{-N} \int_{0}^{\infty} e^{y} P\left(\sup _{0 \leq p_{i} \leq T} Y\left(p_{1}, \cdots, p_{N}\right)>y\right) d y
$$

and $0<H_{a}<\infty$, where $\psi(x)=(2 \pi)^{-1} x^{-1} \exp \left(-x^{2} / 2\right)$, and $\{Y(p)\}$ is a nonbomogeneous Gaussian process with $Y(0, \cdots, 0)=0, E Y(p)=-|p|^{\alpha} / 2$ and $\operatorname{Cov}(Y(p), Y(q))=\left(|p|^{\alpha}+|q|^{a}-|p-q|^{\alpha}\right) / 2$. 
In order to prove this theorem, we first establish the following lemmas. Define $c(x)=\left(\tilde{\sigma}^{-1}(1 / x)\right)^{-1}$ for large $x$. Now by stationarity, we may assume $D \subset \Pi_{i=1}^{N}[0, t]$ for some $t>0$.

Lemma 2.1. Let $X$ satisfy the conditions in Theorem 2.1. Then for any $a>0$, we have

$$
\begin{aligned}
\lim _{x \rightarrow \infty} & \frac{P\left(Z\left(I_{x}[a \mathbf{n} / c(x)]\right)>x\right)}{\psi(x)}=H_{\alpha}(\mathbf{n}, a) \\
& \equiv 1+\int_{0}^{\infty} e^{y} P\left(\max _{0 \leq k_{i} \leq n ; \mathbf{k} \neq 0} Y(a \mathbf{k})>y\right) d y<\infty,
\end{aligned}
$$

where $\{Y(p)\}$ is described in Theorem 2.1, and

$$
I_{x}[a \mathbf{n} / c(x)]=\left\{p \in R^{N} ; p=a \mathbf{k} / c(x), \mathbf{k}=\left(k_{1}, \cdots, k_{N}\right), 0 \leq k_{i} \leq n, k_{i} \text { integers }\right\} .
$$

Proof.

$$
\begin{aligned}
P\left(Z\left(I_{x}[a \mathrm{n} / c(x)]>x\right)=\right. & P(X(0)>x) \\
& +P\left(X(0) \leq x, \max _{0 \leq k_{i} \leq n ; \mathbf{k} \neq 0} X(a \mathbf{k} / c(x))>x\right) .
\end{aligned}
$$

The second term is equal to

$$
\int_{-\infty}^{x} P\left(\max _{0 \leq k_{i} \leq n ; \mathrm{k} \neq 0} X(a \mathrm{k} / c(x))>x \mid X(0)=u\right) \phi(u) d u,
$$

where $\phi(u)$ is the standard normal density. Substituting $u=x-y / x$ and defining $Y_{1}(p)=x(X(p / c(x))-x)+y$, the second term becomes

$$
\begin{aligned}
& \psi(x) \int_{0}^{\infty} e^{y} P\left(\max _{0 \leq k_{i} \leq n ; \mathrm{k} \neq 0} X(a \mathrm{k} / c(x))>y \mid X(0)=x-y / x\right) \exp \left(-y^{2} /\left(2 x^{2}\right)\right) d y \\
& \quad=\psi(x) \int_{0}^{\infty} e^{y} P\left(\max _{0 \leq k_{i} \leq n ; \mathrm{k} \neq 0} Y_{1}(a \mathrm{k})>y \mid X(0)=x-y / x\right) \exp \left(-y^{2} /\left(2 x^{2}\right)\right) d y .
\end{aligned}
$$

Now as in Lemma 2.2 of [4], we can obtain that

$$
\begin{aligned}
E\left(Y_{1}(p) \mid X(0)=x-y / x\right)=x\left\{\rho\left(\frac{1}{c(x)}|p|\right)\left(x-\frac{y}{x}\right)-x\right\}+y \\
=-x^{2}\left\{1-\rho\left(\frac{1}{c(x)}|p|\right)\right\}+y\left\{1-\rho\left(\frac{1}{c(x)}|p|\right)\right\} \\
=-x^{2} \tilde{\sigma}^{2}\left(\frac{1}{c(x)}\right) \frac{|p|^{a}}{2}+o(1)=-\frac{|p|^{a}}{2}+o(1) \text { as } x \rightarrow \infty,
\end{aligned}
$$

and 


$$
\begin{aligned}
& \operatorname{Cov}\left(Y_{1}(p), Y_{1}(q) \mid X(0)=x-y / x\right)=x^{2}\left\{\rho\left(\frac{1}{c(x)}|p-q|\right)-\rho\left(\frac{1}{c(x)}|p|\right) \rho\left(\frac{1}{c(x)}|q|\right)\right\} \\
& =\frac{x^{2}}{2}\left\{-\tilde{\sigma}^{2}\left(\frac{1}{c(x)}\right)|p-q|^{\alpha}+\tilde{\sigma}^{2}\left(\frac{1}{c(x)}\right)|p|^{\alpha}+\tilde{\sigma}^{2}\left(\frac{1}{c(x)}\right)|q|^{\alpha}-\tilde{\sigma}^{4}\left(\frac{1}{c(x)}\right) \frac{|p|^{\alpha}|q|^{\alpha}}{2}\right\}+o(1) \\
& =1 / 2\left\{-|p-q|^{\alpha}+|p|^{\alpha}+|q|^{\alpha}\right\}+o(1) \quad \text { as } x \rightarrow \infty .
\end{aligned}
$$

Consequently,

$$
P\left(\max _{0 \leq k_{i} \leq n ; \mathbf{k} \neq 0} Y_{1}(a \mathbf{k})>y \mid X(0)=x-y / x\right) \rightarrow P\left(\max _{0 \leq k_{i} \leq n ; \mathbf{k} \neq 0} Y(a \mathbf{k})>y\right) \text { as } x \rightarrow \infty .
$$

In order to see that the Lebesgue dominated convergence theorem completes the proof of Lemma 2.1, apply Boole's inequality to the left-hand side of the previous line to obtain a finite number of terms each dominated by an integrable function.

Corollary to Lemma 2.1. Let $X$ satisfy the same conditions as in Theorem 2.1 , except that $\lim _{s \rightarrow 0} \sigma^{2}(s) / \tilde{\sigma}^{2}(s)=C>0$. Then for any $a>0$,

$$
\lim _{x \rightarrow \infty} \frac{P\left(Z\left(I_{x}\left[a_{\mathrm{n}} / c(x)\right]\right)>x\right)}{\psi(x)}=H_{a}\left(\mathrm{n}, C^{1 / a_{a}}\right) .
$$

Proof. We have for the corresponding $Y_{1}$ in Lemma 2.1,

and

$$
E\left(Y_{1}(p) \mid X(0)=x-y / x\right) \rightarrow C|p|^{a} / 2 \text { as } x \rightarrow \infty
$$

$$
\operatorname{Cov}\left(Y_{1}(p), Y_{1}(q) \mid X(0)=x-y / x\right) \rightarrow C\left(|p|^{\alpha}+|q|^{\alpha}-|p-q|^{\alpha}\right) / 2 \text { as } x \rightarrow \infty .
$$

Now, set $\tilde{Y}\left(t_{1} \cdots t_{N}\right)=Y\left(C^{1 / a_{t_{1}}}, \cdots, C^{1 / a_{t_{N}}}\right)$, where $Y$ is the process defined in Theorem 2.1. Then

$$
\begin{array}{r}
P\left(\max _{0 \leq k_{i} \leq n ; \mathbf{k} \neq 0} Y_{1}(a \mathbf{k})>y \mid X(0)=x-y / x\right) \rightarrow P\left(\max _{0 \leq k_{i} \leq n ; \mathbf{k} \neq 0} \tilde{Y}(a \mathbf{k})>y\right) \\
=P\left(\operatorname { m a x } _ { 0 \leq k _ { i } \leq n ; \mathbf { k } \neq 0 } Y \left(C^{\left.\left.1 / a_{a \mathbf{k}}\right)>y\right)} \text { as } x \rightarrow \infty .\right.\right.
\end{array}
$$

Lemma 2.2. Let $X$ satisfy the conditions in Theorem 2.1. For any $a>0$,

$$
\varliminf_{x \rightarrow \infty} \frac{P\left(Z\left(D_{(x)}\right)>x\right)}{\mu(D) \psi(x)_{c}(x)^{N}} \geq a^{-N}\left(1-2^{N} \Sigma^{*}\left(1-\Phi\left(2^{-1}(|\mathbf{k}| a)^{a / 2}\right)\right)\right),
$$

where $D_{(x)}=\left\{p: p=a \mathbf{k} / c(x) \in D, k_{i}\right.$ integers $\}$ and $\Sigma^{*}$ denotes the $N$-fold sum over $0 \leq k_{i}<\infty(i=1, \cdots, N)$ but $\mathbf{k} \neq \mathbf{0}$.

Proof. Denoting the event $B_{k}=\{X(a k / c(x))>x\}$ and using stationarity we have 


$$
\begin{aligned}
& P\left(Z_{i}\left(D_{(x)}\right)>x\right) \geq P\left(\bigcup_{\mathbf{k} \in \tilde{\mathbf{\Lambda}}} B_{\mathbf{k}}\right) \\
& \geq \sum \underset{\mathbf{k} \in \overline{\boldsymbol{\Lambda}}}{\cdots} \sum P\left(B_{\mathbf{k}}\right)-1 / 2 \sum_{\mathbf{k} \neq l ; \mathbf{k}, \boldsymbol{l} \in \overline{\mathbf{\Lambda}}} \cdots \sum P\left(B_{\mathbf{k}} \cap B_{\boldsymbol{l}}\right) \\
& \geq \#(\widetilde{\Lambda})\left(P\left(B_{0}\right)-2^{N-1} \sum_{0 \leq k_{i<m ;}} \cdots \sum_{\mathbf{k} \neq 0} P\left(B_{0} \cap B_{\mathbf{k}}\right)\right) \text {, }
\end{aligned}
$$

where $\tilde{\Lambda}=\left\{\mathbf{k} \in R^{N}: I_{a, x}(\mathbf{k}) \subset D\right\}, \#(\tilde{\Lambda})=$ the cardinal number of $\tilde{\Lambda}$,

$$
I_{a, x}(\mathbf{k})=\left\{p \in R^{N}: a k_{i} / c(x) \leq p_{i} \leq a\left(k_{i}+1\right) / c(x), i=1, \cdots, N\right\},
$$

$m=[t c(x) / a]+1$, and [ ] denotes the greatest integer function.

We recall the result of Pickands [2, Lemma 2.3],

$$
P\left(B_{0} \cap B_{k}\right) \leq 2 \psi(x)\left\{1-\Phi\left(x(1-\rho)^{1 / 2}(1+\rho)^{-1 / 2}\right)\right\},
$$

where $\rho=\rho(a|\mathbf{k}| / c(x))$ is the correlation coefficient for $X(\mathbf{0})$ and $X(a \mathbf{k} / c(x))$ and $\Phi$ is the standard normal distribution function and $|\mathbf{k}|=\sqrt{k_{1}^{2}+k_{2}^{2}+\cdots+k_{N}^{2}}$. Now we note that

$$
\#(\Lambda) / \mu(D)\left(\tilde{\sigma}^{-1}(1 / x)\right)^{-N}=\mu\left(\bigcup_{\mathbf{k} \in \tilde{\Lambda}} I_{a, x}(\mathbf{k})\right) / \mu(D) a^{N} \rightarrow a^{-N} \quad \text { as } x \rightarrow \infty,
$$

since $\mu(\partial D)=0$. Therefore we have

$\frac{\lim }{x \rightarrow \infty} \frac{P\left(Z\left(D_{(x)}\right)>x\right)}{\mu(D) \psi(x)(c(x))^{N}} \geq a^{-N}\left\{1-2^{N} \varlimsup_{x \rightarrow \infty} \sum_{0 \leq k} \cdots \sum_{i \leq m}\left\{1-\Phi\left(x(1-\rho)^{1 / 2}(1+\rho)^{-1 / 2}\right)\right\}\right\}$.

To study the lim sup of the above sum, we partition the sum into three parts according to (i) $|\mathbf{k}| a \leq t_{0}$, (ii) $|\mathbf{k}| a>t_{0},|\mathbf{k}| a / c(x)<\delta$, and (iii) $|\mathbf{k}| a>t_{0}$, $|\mathbf{k}| a / c(x) \geq \delta$, where the values of $\delta>0$ and $t_{0}>1$ will be selected later.

Since the number of terms in $\Sigma^{(i)}$ is finite, we have

$$
\lim _{x \rightarrow \infty} \sum^{(i)}(1-\Phi)=\sum^{(i)} \lim _{x \rightarrow \infty}(1-\Phi) .
$$

We may ignore the third sum $\Sigma^{(\mathrm{iii})}$. For $|\mathbf{k}| a / c(x) \geq \delta$, there exists a constant $\kappa$ such that $1-\rho \geq \kappa>0$, and

$$
\begin{aligned}
\sum^{(\mathrm{iii})}\left\{1-\Phi\left(x\left(\frac{1-\rho}{1+\rho}\right)^{1 / 2}\right)\right\} & \leq \sum^{(\mathrm{iii})}\left(1-\Phi\left(x(\kappa / 2)^{1 / 2}\right)\right) \leq m^{N} \psi\left(x(\kappa / 2)^{1 / 2}\right) \\
& \leq(t c(x) / a)^{N} \exp \left(-\kappa x^{2} / 2\right) \rightarrow 0 \text { as } x \rightarrow \infty .
\end{aligned}
$$

Now for $\Delta_{1}$ sufficiently small, we have $A_{1}\left(\Delta_{1}\right)>0$. Then for all the terms $\Sigma^{(\mathrm{ii})}$ when $|\mathbf{k}| a / c(x)<\Delta_{1}$, we have 


$$
\begin{aligned}
x\left(\frac{1-\rho}{1+\rho}\right)^{1 / 2} & \geq x \sigma(|\mathbf{k}| a / c(x))=\frac{1}{2} \frac{\sigma(|\mathbf{k}| a / c(x))}{\tilde{\sigma}(1 / c(x))} \\
& \geq \frac{A_{1}\left(\Delta_{1}\right)}{2}-\frac{\tilde{\sigma}(|\mathbf{k}| a / c(x))}{\tilde{\sigma}(1 / c(x))}=\frac{A_{1}\left(\Delta_{1}\right)}{2}(|\mathbf{k}| a)^{a / 2}\left(\frac{H(|\mathbf{k}| a / c(x))}{H(1 / c(x))}\right)^{1 / 2} .
\end{aligned}
$$

By property (2.5), there exists a $\delta_{\alpha}>0$ such that $H(|\mathbf{k}| a / c(x)) / H(1 / c(x)) \geq$ $(|\mathbf{k}| a)^{-a / 4}$, provided $|\mathbf{k}| a / c(x)<\delta_{a}$, and $|\mathbf{k}| a>t_{0}>1$. Now for the definition of $\Sigma^{(i i)}$, take $\delta=\min \left(\delta_{a}, \Delta_{1}\right)$. Then

$$
\inf _{T \leq x \leq \infty} x\left(\frac{1-\rho}{1+\rho}\right)^{1 / 2} \geq \frac{A_{1}(\delta)}{2}(|\mathbf{k}| a)^{a / 4},
$$

provided $|\mathbf{k}| a / c(x)<\delta,|\mathbf{k}| a>t_{0}$ and $T$ is large.

Finally, defining $a_{\mathbf{k}}(x)=\left\{1-\Phi\left(x(1-\rho)^{1 / 2}(1+\rho)^{-1 / 2}\right)\right\}$ for $|\mathbf{k}| a / c(x)<\delta$ and $a_{\mathbf{k}}(x)=\left\{1-\Phi\left(2^{-1} A_{1}(\delta)(|\mathbf{k}| a)^{a / 4}\right)\right\}$ for $|\mathbf{k}| a / c(x) \geq \delta$, we have

$$
\sum^{*} \sup _{T \leq x<\infty} a_{k}(x) \leq \sum^{*}\left\{1-\Phi\left(2^{-1} A_{1}(\delta)(|\mathbf{k}| a)^{a / 4}\right)\right\}<\infty .
$$

Therefore, it follows that

$$
\begin{aligned}
\varlimsup_{x \rightarrow \infty} \sum_{0 \leq k_{i}<m ;} \ldots \sum_{\mathbf{k} \neq 0} a_{\mathbf{k}}(x) & \leq \sum^{*} \varlimsup_{x \rightarrow \infty} a_{\mathbf{k}}(x) \\
& =\sum^{*}\left\{1-\Phi\left(2^{-1}(|\mathbf{k}| a)^{a / 2}\right)\right\}<\infty,
\end{aligned}
$$

since

$$
x(1-\rho)^{1 / 2}(1+\rho)^{-1 / 2} \sim \frac{x}{2} \cdot \sigma(|\mathbf{k}| a / c(x)) \sim \frac{1}{2} \frac{\tilde{\sigma}(|\mathbf{k}| a / c(x))}{\tilde{\sigma}(1 / c(x))} \rightarrow \frac{1}{2}(|\mathbf{k}| a)^{a / 2} \text { as } x \rightarrow \infty .
$$

Lemma 2.2 now follows.

Corollary to Lemma 2.2. For $X$ satisfying the same conditions as in Theorem 2.1 , except that $\lim _{s \rightarrow 0} \sigma^{2}(s) / \tilde{\sigma}^{2}(s)=C>0$, we have

$$
\lim _{x \rightarrow \infty} \frac{P\left(Z\left(D_{(x)}\right)>x\right)}{\mu(D) \psi(x)\left(\tilde{\sigma}^{-1}(1 / x)\right)^{-N}} \geq a^{-N}\left(1-2^{N} \Sigma^{*}\left(1-\Phi\left(2^{-1}\left(|\mathbf{k}| C^{1 / a_{a}}\right)^{\alpha / 2}\right)\right)\right) .
$$

Proof. We have only to note that

$$
x(1-\rho)^{1 / 2}(1+\rho)^{-1 / 2} \rightarrow 1 / 2(|\mathbf{k}| a)^{a / 2} C^{1 / 2} \text { as } x \rightarrow \infty .
$$

Lemma 2.3. Let $X$ satisfy the conditions in Theorem 2.1. For $a>0$,

$$
\lim _{x \rightarrow \infty} \frac{P\left(z\left(D_{(x)}\right)>x\right)}{\mu(D) \psi(x)(c(x))^{N}}=\frac{H_{a}(a)}{a^{N}}
$$


where $0<H_{a}(a) \equiv \lim _{n \rightarrow \infty}\left(H d_{d}(\mathbf{n}, a) / n^{N}\right)<\infty$.

Proof. Let $\left(X^{+}\right)^{N}=\left\{\mathbf{k} \in R^{N}: k_{i}\right.$ are nonnegative integers, $\left.i=1, \cdots, N\right\}$. For $\mathbf{k} \in\left(X^{+}\right)^{N}$, let

$$
B_{\mathbf{k}}=\left\{X\left(a \mathbf{k} / c_{1}(x)\right)>x\right\}
$$

and also for arbitrary $n>0$ and $l \in\left(X^{+}\right)^{N}$, let

$$
A_{l}=\bigcup_{j_{1}=\left(l_{1}-1\right) n}^{l_{1} n-1} \bigcup_{j_{2}=\left(l_{2}-1\right) n}^{l_{2} n-1} \cdots \bigcup_{j_{N}=\left(l_{N}-1\right) n}^{l_{N} n-1} B_{i}
$$

By stationarity, $P\left(A_{l}\right)=P\left(A_{i}, \ldots, 1\right)$ for all $l$ with the $l_{i} \geq 1$.

where

$$
P\left(\bigcup_{l \in \Lambda_{1}} A_{l}\right) \leq P\left(Z\left(D_{(x)}\right)>x\right) \leq P\left(\bigcup_{l \in \Lambda_{2}} A_{l}\right),
$$

$$
\Lambda_{1}=\left\{l \in R^{N}: I_{l}^{(n)} \subset D\right\}, \quad \Lambda_{2}=\left\{l \in R^{N}: I_{l}^{(n)} \cap D \neq \varnothing\right\}
$$

and

$$
i_{l}^{(n)}=\left\{p \in R^{N}: a\left(l_{i}-1\right) n / c(x) \leq p_{i} \leq a l_{i} n / c(x), i=1, \cdots, N\right\}
$$

Consequently,

$$
P\left(Z\left(D_{(x)}\right)>x\right) \leq \#\left(\Lambda_{2}\right) P\left(A_{1, \cdots, 1}\right) .
$$

Therefore, using Lemma 2.1, we obtain

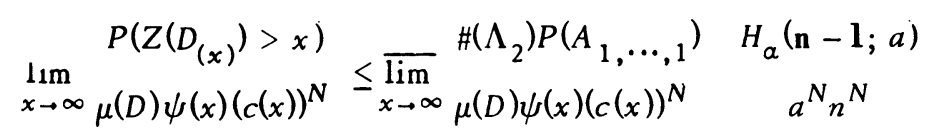

On the other hand, (2.9) and stationarity imply

$$
\begin{aligned}
& \left.P\left(Z\left(D_{(x)}\right)>x\right) \geq \sum \underset{l \in \Lambda_{1}}{\sum \cdots \sum} P\left(A_{l}\right)-\frac{1}{2} \sum_{k \neq l ; k, l \in \Lambda_{1}} \cdots \sum_{k} \cap A_{l}\right) \\
& \geq \#\left(\Lambda_{1}\right)\left\{P\left(A_{1, \cdots, 1}\right)-2^{N-1} \sum_{0 \leq j<<} \cdots \sum_{0 \leq s_{i}<m ; \text { some } s_{i} \geq n} P\left(B_{j} \cap B_{\mathbf{s}}\right)\right\},
\end{aligned}
$$

where $m=\lfloor t c(x) / a\rfloor+1$. We note here that

$$
\#\left(\Lambda_{1}\right) / \mu(D)(c(x))^{N} \rightarrow n^{-N} a^{-N^{\prime}} \quad \text { as } x \rightarrow \infty .
$$

Again using Lemma 2.3 in Pickands [2], we obtain

$$
\left.\begin{array}{r}
\lim _{x \rightarrow \infty} \frac{P\left(Z\left(D_{(x)}\right)>x\right)}{\mu(D) \psi(x)(c(x))^{N}} \geq n^{-N} a^{-N}\left\{H_{a}(\mathbf{n}-1, a)-2^{N-1} \varlimsup_{x \rightarrow \infty} \sum^{* * P\left(B_{\mathbf{j}} \cap B_{\mathbf{s}}\right)}\right. \\
\psi(x)
\end{array}\right\}
$$


where $\rho=\rho(a|\mathbf{j}-\mathbf{s}| / c(x))$ and

$$
\begin{aligned}
\Sigma^{* *}=\sum_{j_{1}=0}^{n-1} \cdots \sum_{j_{N=0}}^{n-1}\left(\sum_{s_{1}=n}^{\infty} \sum_{s_{2}=0}^{\infty} \cdots \sum_{s_{N}=0}^{\infty}+\right. & \sum_{s_{1}=0}^{n-1} \sum_{s_{2}=n}^{\infty} \cdots \sum_{s_{N=0}}^{\infty} \\
& \left.+\cdots+\sum_{s_{1}=0}^{n-1} \cdots \sum_{s_{N-1}=0}^{n-1} \sum_{s_{N}=n}^{\infty}\right) .
\end{aligned}
$$

In the study of $\overline{\lim }_{x \rightarrow \infty} \Sigma^{* *}(1-\Phi)$, we use the same device as in Lemma 2.2 . So we have

$\lim _{x \rightarrow \infty} \frac{P\left(Z\left(D_{(x)}\right)>x\right)}{\mu(D) \psi(x)(c(x))^{N}} \geq n^{-N} a^{-N}\left\{H_{\alpha}(\mathbf{n}-1, a)-2^{N} \Sigma^{* *}\left(1-\Phi\left(2^{-1}(|\mathbf{j}-\mathbf{s}| a)^{a / 2}\right)\right)\right\}$.

Since the left-hand side does not depend on $n$, we have

$$
\lim _{x \rightarrow \infty} \frac{P\left(Z\left(D_{(x)}\right)>x\right)}{\mu(D) \psi(x)(c(x))^{N}} \geq a^{-N} \varlimsup_{n \rightarrow \infty} \frac{H_{a}(\mathbf{n}-1, a)}{n^{N}}-2^{N} \varliminf_{n \rightarrow \infty} \sum^{* *} \frac{d_{\mathrm{j}-\mathbf{s}}}{n^{N}} .
$$

Now Lemma 2.2 implies $\Sigma^{* *} d_{j-s}<\infty$ and then Kronecker's lemma implies

$$
\varliminf_{n \rightarrow \infty} \sum^{* *} d_{\mathrm{j}-\mathrm{s}} / n^{N}=0
$$

Combining (2.10) and (2.12) yields

$$
\begin{aligned}
a^{-N} \varlimsup_{n \rightarrow \infty} \frac{H_{a}(\mathbf{n}-1, a)}{n^{N}} \leq \varliminf_{x \rightarrow \infty} \frac{P\left(Z\left(D_{(x)}\right)>x\right)}{\mu(D) \psi(x)(c(x))^{N}} \\
\quad \leq \varlimsup_{x \rightarrow \infty} \frac{P\left(Z\left(D_{(x)}\right)>x\right)}{\mu(D) \psi(x)(c(x))^{N}} \leq a^{-N} \frac{\lim _{n \rightarrow \infty}}{\frac{H_{a}(\mathbf{n}-1, a)}{n^{N}} .}
\end{aligned}
$$

This establishes that $\lim _{n \rightarrow \infty}\left(H_{a}(\mathbf{n}, a) / n^{N}\right)=H_{a}(a)$ exists and that

$$
\lim _{x \rightarrow \infty} \frac{P\left(Z\left(D_{(x)}\right)>x\right)}{\mu(D) \psi(x)(c(x))^{N}}=\frac{H_{a}(a)}{a^{N}} .
$$

Now (2.10) implies $H_{a}(a)<\infty$. Since the right-hand side of (2.8) in Lemma 2.3 is positive for all sufficiently large $a$, we know $H_{a}(a)>0$ for all $a>a_{0}$, say. For arbitrary $a>0$, we select an integer $m$ such that $m a>a_{0}$. Then $H_{a}(\mathbf{n}, a m) \leq$ $H_{a}(\mathbf{n} m, a)$ implies $0<H_{a}(a m) \leq m^{N} H_{a}(a)$. So $H_{a}(a)>0$ for all $a>0$.

Corollary to Lemma 2.3. Let $X$ be a continuous Gaussian random field with mean zero and variance one satisfying condition (1.1) for all $p, q \in D$ with $|p-q|<\delta$. Then

$$
\begin{aligned}
\frac{H_{a}\left(C_{1}^{* 1 / a_{a}}\right)}{a^{N}} & \leq \lim _{x \rightarrow \infty} \frac{P\left(Z\left(D_{(x)}\right)>x\right)}{\mu(D) \psi(x)(c(x))^{N}} \\
& \leq \varlimsup_{x \rightarrow \infty} \frac{P\left(Z\left(D_{(x)}\right)>x\right)}{\mu(D) \psi(x)(c(x))^{N}} \leq \frac{H_{a}\left(C_{2}^{* 1 / a_{a}}\right)}{a^{N}}
\end{aligned}
$$


for any $C_{1}^{*}, C_{2}^{*}$ such that $0<C_{1}^{*}<C_{1}<C_{2}<C_{2}^{*}$.

Proof. Define two stationary isotropic Gaussian processes $X^{(1)}, X^{(2)}$ having $\sigma_{i}^{2}(|p-q|) \equiv E\left(\left|X^{(i)}(p)-X^{(i)}(q)\right|^{2}\right)=2 C_{i}^{*}|p-q|^{\alpha} H(|p-q|)+o\left(|p-q|^{\alpha} H(|p-q|)\right)$ as $|p-q| \rightarrow 0$. We use the label $(i)$ in this proof to denote expressions involving the process $X^{(i)}$.

By hypothesis, we then have

$$
\sigma_{1}^{2}(|p-q|) \leq 2 C_{1} \widetilde{\sigma}^{2}(|p-q|) \leq \sigma^{2}(p, q) \leq 2 C_{2} \tilde{\sigma}^{2}(|p-q|) \leq \sigma_{2}^{2}(|p-q|)
$$

for all $p, q \in I_{l}^{(n)}$ (when $x$ is sufficiently large) and uniformly for $l \in \Lambda_{2}$. So, using Slepian's result [5], we have

$$
P\left(A_{l}\right) \leq P\left(A_{l}^{(2)}\right) \text { for large } x,
$$

where $A_{1, \ldots, 1}^{(2)}$ is the event $A_{1, \ldots, 1}$ defined in terms of the process $X^{(2)}$. Now

$$
\varlimsup_{x \rightarrow \infty} \frac{P\left(Z\left(D_{(x)}\right)>x\right)}{\mu(D) \psi(x)(c(x))^{N}} \leq \varlimsup_{x \rightarrow \infty} \frac{\#\left(\Lambda_{2}\right) P\left(A_{l}^{(2)}\right)}{\mu(D) \psi(x)(c(x))^{N}}=\frac{H_{a}\left(\mathrm{n}, C_{2}^{\left.* 1 / a_{a}\right)}\right.}{a^{N} n^{N}},
$$

and also

$$
P\left(\bigcup_{l \in \Lambda_{1}} A_{l}\right) \geq \#\left(\Lambda_{1}\right) P\left(A_{l}^{(1)}\right)-\frac{1}{2} \sum_{\mathbf{k} \neq l}^{\cdots} \sum P\left(A_{\mathbf{k}} \cap A_{l}\right) .
$$

As in the proof of Lemma 2.3, the sum $\Sigma P\left(A_{\mathbf{k}} \cap A_{l}\right)$ can be bounded above by a sum $\Sigma P\left(B_{i} \cap B_{i}\right)$. But

$$
\begin{aligned}
P\left(B_{\mathbf{i}} \cap B_{\mathbf{j}}\right) & \left.=P\left(B_{\mathbf{i}}^{(1)}\right)+P\left(B_{\mathbf{j}}^{\prime}\right)\right)-P\left(B_{\mathbf{i}} \cup B_{\mathbf{j}}\right) \\
& \leq P\left(B_{\mathbf{i}}^{(1)}\right)+P\left(B_{\mathbf{j}}^{(1)}\right)-P\left(B_{\mathbf{i}}^{(1)} \cup B_{\mathbf{j}}^{(1)}\right)=P\left(B_{\mathbf{i}}^{(1)} \cap B_{\mathbf{i}}^{(1)}\right) .
\end{aligned}
$$

Consequently, as in the proof of Lemma 2.3, the sum $\Sigma P\left(A_{k} \cap A_{l}\right)$ can eventually be ignored (as $n \rightarrow \infty$ ). So

$$
\lim _{n \rightarrow \infty} \frac{H_{a}\left(\mathbf{n}, C_{1}^{\left.* 1 / a_{a}\right)}\right.}{a^{N} n^{N}} \leq \frac{\lim }{x \rightarrow \infty} \frac{P\left(Z\left(D_{(x)}\right)>x\right)}{\mu(D) \psi(x)(c(x))^{N}} .
$$

From (2.14) and (2.15) follows (2.13).

Lemma 2.4. Under the same assumptions as in Theorem 2.1, we have, for any $a>0,2^{-a / 4}<b<1$, and $\gamma>0$,

where

$$
\varlimsup_{x \rightarrow \infty} \frac{P(X(0)<x-\gamma / x, Z(I[\mathbf{a} / c(x-\gamma / x)]>x)}{\psi(x)} \leq M(a, \gamma)
$$

$$
\begin{aligned}
& I\lfloor\mathbf{a} / c(x-y / x)\}=\left\{p \in R^{N}: 0 \leq p_{i} \leq a / c(x-\gamma / x)\right\}, \\
& M(a, \gamma)=(\sqrt{N} a / 2)^{a / 2} 2^{N} \sum_{k=0}^{\infty} 2^{k(N-\alpha / 2)} R\left(\gamma(1-b)(2 / a \sqrt{N})^{a / 2}\left(2^{\alpha / 2} b\right)^{k}\right. \\
& \left.-2^{-1}(a \sqrt{N} / 2)^{a / 2} 2^{-a k / 2}\right),
\end{aligned}
$$


and

$$
R(x)=\int_{x}^{\infty}(1-\Phi(s)) d s
$$

Proof. We note that

$$
\{X(\mathbf{0}) \leq x-\gamma / \dot{x}, Z(I[\mathrm{a} / c(x-\gamma / x)])>x\} \subseteq \bigcup_{k=0}^{\infty} D_{k},
$$

where

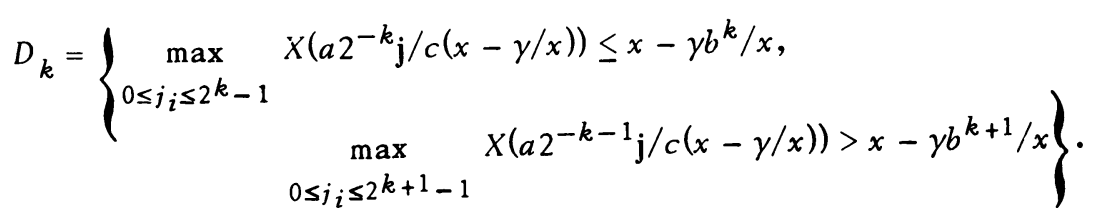

Also, $D_{k} \subseteq \bigcup_{0 \leq j_{i} \leq 2^{k-1}} E_{\mathbf{j}, k}$ where

$$
\begin{gathered}
E_{\mathbf{j}, k}=\left\{X\left(a 2^{-k} \mathbf{j} / c(x-\gamma / x)\right) \leq x-\gamma b^{k} / x,\right. \\
\max _{2 j_{i} \leq l_{i} \leq 2 j_{i}+1} X\left(a 2^{-k} l / c(x-\gamma / x)\right)>x-\gamma b^{k+1} / x
\end{gathered} .
$$

By using [4, Lemma 2.6], we obtain

$$
P\left(E_{\mathbf{j}, k}\right) \leq \sum_{\epsilon \neq 0} \rho^{-1}\left(1-\rho^{2}\right)^{1 / 2} \psi(x) R(y),
$$

where $\rho=\rho\left(a 2^{-k-1} \sqrt{\epsilon_{1}^{2}+\cdots+\epsilon_{N}^{2}} / c(x-\gamma / x)\right), \Sigma_{\epsilon \neq 0}$ is a sum over the set $\left\{\epsilon \neq 0: \epsilon_{j}=0\right.$ or 1$\}$, and $y=y(x)=\gamma(1-b) b^{k} \rho x^{-1}\left(1-\rho^{2}\right)^{-1 / 2}-x(1+p)^{-1}\left(1-\rho^{2}\right)^{1 / 2}$. Consequently,

$$
\begin{aligned}
\varlimsup_{x \rightarrow \infty} & P\{X(0) \leq x-\gamma / x, Z(I[\mathbf{a} / c(x-y / x)])>x\} \\
& \leq \overline{\lim }_{x \rightarrow \infty} \sum_{k=0}^{\infty} \sum_{0 \leq j_{i} \leq 2^{k}-1} P\left(E_{j, k}\right) / \psi(x) \\
& \leq \varlimsup_{x \rightarrow \infty} \sum_{k=0}^{\infty} 2^{N k} \sum_{\in \neq 0} \rho^{-1}\left(1-\rho^{2}\right)^{1 / 2} R(y(x))
\end{aligned}
$$

By using property (2.6) when $k$ is sufficiently large, we can obtain the following estimates for large $x$,

and

$$
x \rho^{-1}\left(1-\rho^{2}\right)^{1 / 2} \leq 2 S^{-1} A_{2} \cdot\left(\sqrt{N} a 2^{-k-1}\right)^{a / 4}
$$

$y=y(x) \geq 2^{-1}\left\{\gamma(1-b) b^{k} S A_{2}^{-1}\left(2^{k+1} / a \sqrt{N}\right)^{a / 4}-4(1+S)^{-1} A_{2}\left(2^{k+1} / a \sqrt{N}\right)^{-\alpha / 4}\right\}$,

where $S=\inf _{0 \leq s \leq a} \sqrt{N} / c(x-\gamma / x) \rho(s)$ and $A_{2}=A_{2}(a \sqrt{N} / c(x-\gamma / x))$. From the above estimate, we can prove 
Therefore

$$
\sum_{k=0}^{\infty} 2^{N k} \sup _{T \leq x<\infty} \sum_{\in \neq 0} \rho^{-1}\left(1-\rho^{2}\right)^{1 / 2} R(y(x))<\infty .
$$

$$
\begin{aligned}
\varlimsup_{x \rightarrow \infty} & \frac{P(X(0) \leq x-\gamma / x, Z(I[\mathrm{a} / c(x-y / x)])>x)}{\psi(x)} \\
& \leq \sum_{k=0}^{\infty} \varlimsup_{x \rightarrow \infty} 2^{N k} \sum_{\epsilon \neq 0} x \rho^{-1}\left(1-\rho^{2}\right)^{1 / 2} R(y(x)) \\
& =\sum_{k=0}^{\infty} 2^{N k} \sum_{\epsilon \neq 0}\left(a|\epsilon| 2^{-k-1}\right)^{\alpha / 2} R\left(\gamma(1-b) b^{k}\left(a|\epsilon| 2^{-k-1}\right)^{-a / 2}-2^{-1}\left(a|\epsilon| 2^{-k-1}\right)^{a / 2}\right) \\
& <M(a, \gamma) .
\end{aligned}
$$

Proof of Theorem 2.1. Define

$$
H_{a}^{+}=\varlimsup_{x \rightarrow \infty} \frac{P(Z(D)>x)}{\mu(D) \psi(x)(c(x))^{N}}
$$

and

$$
H_{a}^{-}=\lim _{x \rightarrow \infty} \frac{P(Z(D)>x)}{\mu(D) \psi(x)(c(x))^{N}}
$$

Now, since $c(x-\gamma / x) / c(x) \rightarrow 1$ and $\psi(x-\gamma / x) / \psi(x)=e^{\gamma}$ as $x \rightarrow \infty$ for $\gamma>0$, we see from Lemma 2.3 that

For $\gamma>0$, we obtain

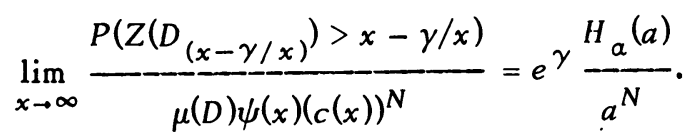

$$
\begin{aligned}
H_{a}^{-}-e^{\gamma} \frac{H_{a}(a)}{a^{N}} \leq H_{a}^{+}-e^{\gamma} \frac{H_{a}^{(a)}}{a^{N}} \\
=\varlimsup_{x \rightarrow \infty} \frac{P(Z(D)>x)-P(Z(D(x-\gamma / x)>x-\gamma / x)}{\mu(D) \psi(x)(c(x))^{N}} \\
=\varlimsup_{x \rightarrow \infty} \frac{P(Z(D)>x, Z(D(x-\gamma / x) \leq x-\gamma / x)}{\mu(D) \psi(x)(c(x))^{N}} \\
\leq \varlimsup_{x \rightarrow \infty} \frac{\#\left(\Lambda_{2}\right) P(X(0) \leq x-\gamma / x, Z(I[a / c(x-\gamma / x)])>x)}{\mu(D) \psi(x)(c(x))^{N}} \\
\leq \frac{1}{a^{N} \varlimsup_{x \rightarrow \infty}} \frac{P(X(0) \leq x-\gamma / x, Z(I[a / c(x-\gamma / x)])>x)}{\psi(x)} \\
\leq \frac{M(a, \gamma)}{a^{N}} .
\end{aligned}
$$


Therefore

$$
0 \leq H_{a}^{-}-\frac{H_{a}(a)}{a^{N}} \leq H_{a}^{+}-\frac{H_{a}(a)}{a^{N}} \leq \frac{M(a, \gamma)}{a^{N}}+\left(e^{\gamma}-1\right) \frac{H_{a}(a)}{a^{N}} .
$$

Since $H_{a}^{-}$and $H_{a}^{+}$are finite, we can see that the $\lim$ sup and $\lim$ inf of $H_{a}(a) / a^{N}$ as $a \rightarrow 0$ must be finite. Now choosing $\gamma=a^{\beta}$ with $0<\beta<\alpha / 2$ in (2.16), we have $M(a, \gamma) / a^{N} \rightarrow 0$ as $a \rightarrow 0$ and $H_{a} \equiv H_{a}^{+}=H_{a}^{-}=\lim _{a \rightarrow 0} H_{a}(a) / a^{N}$.

In a manner similar to the proof of Theorem 2.1 in [4], we can show

$$
H_{a}=\lim _{T \rightarrow \infty} T^{-N}\left(1+\int_{0}^{\infty} e^{s P}\left(\sup _{0 \leq t_{i} \leq T} Y(\mathbf{t})>s\right) d s\right) .
$$

Corollary to Theorem 2.1. Let $X$ be a continuous Gaussian random field with mean zero and variance one satisfying condition (1.1) for all $p, q \in D$ with $|p-q|<\delta$. Then

$$
H_{a} C_{1}^{N / a} \leq \lim _{x \rightarrow \infty} \frac{P(Z(D)>x)}{\mu(D) \psi(x)(c(x))^{N}} \leq \varlimsup_{x \rightarrow \infty} \frac{P(Z(D)>x)}{\mu(D) \psi(x)(c(x))^{N}} \leq H_{a} C_{2}^{N / a} .
$$

Proof. If $X$ satisfies the conditions of the Corollary to Lemma 2.1, then note that

$$
\lim _{x \rightarrow \infty} \frac{P(Z(D)>x)}{\mu(D) \psi(x)(c(x))^{N}}=\lim _{a \rightarrow 0} \frac{H_{a}\left(C^{1 / a_{a}}\right)}{a^{N}}=C^{N / a} H_{a} .
$$

Now let $D \subset \Pi_{i=1}^{N}[0, t]$, let $l$ be an arbitrary number $<\delta / \sqrt{N}$, and let the rectangle $I_{\mathbf{k}}^{l}=\left\{p \in R^{N}: l \cdot k_{j} \leq p_{j} \leq l\left(k_{j}+1\right)\right\}$. Let $K=\left\{\mathbf{k}: I_{\mathbf{k}}^{l} \cap D \neq \varnothing\right\}$. Then by the use of Slepian's theorem, we have

$$
P(Z(D)>x) \leq \sum_{\mathbf{k} \in K} P\left(Z\left(I_{\mathbf{k}}^{l}\right)>x\right) \leq \#(K) P\left(Z^{(2)}\left(I_{0}^{l}\right)>x\right),
$$

where $Z^{(2)}(A)=\max _{p \in A} X^{(2)}(p)$ and $X^{(2)}$ is a process defined in the proof of the Corollary to Lemma 2.3. Therefore

$$
\begin{aligned}
& \varlimsup_{x \rightarrow \infty} \frac{P(Z(D)>x)}{\mu(D) \psi(x)(c(x))^{N}} \leq \frac{\#(K) l^{N}}{\mu(D)} \varlimsup_{x \rightarrow \infty} \frac{P\left(Z^{(2)}\left(I_{0}^{l}\right)>x\right)}{\mu\left(I_{0}^{l}\right) \psi(x)(c(x))^{N}} \\
& =\frac{\#(K) l^{N}}{\mu(D)} C_{2}^{* N / a} H_{a} \rightarrow C_{2}^{* N / a} H_{a} \text { as } l \rightarrow 0 .
\end{aligned}
$$

Since $C_{2}^{*}>C_{2}$ is arbitrary, we have the right-hand side of (2.17).

Now by the Corollary to Lemma 2.3 ,

$$
\begin{gathered}
\lim _{x \rightarrow \infty} \frac{P(Z(D)>x)}{\mu(D) \psi(x)(c(x))^{N}} \geq \lim _{x \rightarrow \infty} \frac{P\left(Z^{(1)}(D(x)>x)\right.}{\mu(D) \psi(x)(c(x))^{N}} \\
\quad \frac{H_{a}\left(C_{1}^{* 1 / a} a\right)}{a^{N}} \rightarrow C_{1}^{* N / a_{a}} H_{a} \text { as } a \rightarrow 0 .
\end{gathered}
$$


Since $C_{1}^{*}<C_{1}$ is arbitrary, we have the left-hand side of (2.17).

3. An asymptotic $0-1$ behavior. In this section we will consider the $\omega$-set

$$
A_{\phi}=\left\{\exists r_{0}: X(p) \leq \phi(|p|) \text { for all }|p| \geq r_{0}\right\}
$$

for an arbitrary nondecreasing function $\phi$. Since $X$ is a continuous Gaussian random field, $A_{\phi}$ is an event. In earlier papers [4] and [5], the authors give a criterion in terms of $\phi$ for deciding whether the $P\left(A_{\phi}\right)$ is 0 or 1 for processes with an $N=1$ dimensional parameter space. Here this criterion is generalized to random fields defined on $R^{N}$ with $N>1$. The proofs are based on the corollaries to Lemma 2.3 and Theorem 2.1 in $\$ 2$.

Theorem 3.1. Suppose $X$ is a continuous Gaussian random field with mean zero and variance one satisfying part (A) of condition (1.1); that is, the correlation function $\rho(p, q)$ satisfies

$$
1-C_{2}|p-q|^{a} H(|p-q|) \leq \rho(p, q),
$$

for all $|p-q|<$ some $\delta_{1},|p|$ and $|q| \geq$ some $T_{1}$, and some $C_{2}>0$. If for any positive nondecreasing function $\phi(r)$,

$$
\int^{\infty}\left(\tilde{\sigma}^{-1}(1 / \phi(r))\right)^{-N}(\phi(r))^{-1} \exp \left(-1 / 2 \phi^{2}(r)\right) r^{N-1} d r<\infty,
$$

then

$$
P\left(A_{\phi}\right)=1
$$

Proof. Let $r_{n}=n \Delta$, where $\Delta>0$ and $n=1,2, \ldots$. From (2.17) we have, for $n$ sufficiently large,

$$
\begin{aligned}
P\left(\max _{r_{n} \leq|p| \leq r_{n+1}} X(p) \geq \phi\left(r_{n}\right)\right) \leq & \operatorname{const} \mu\left(K_{n}\right)\left(\phi\left(r_{n}\right)\right)^{-1}\left(\tilde{\sigma}^{-1}\left(1 / \phi\left(r_{n}\right)\right)\right)^{-N} \\
& \cdot \exp \left(-1 / 2 \phi^{2}\left(r_{n}\right)\right),
\end{aligned}
$$

where $K_{n}=\left\{p: r_{n} \leq|p| \leq r_{n+1}\right\}$. The convergence of the right-hand side of (2.17) can be shown to be uniform with respect to all the sets $K_{n}$; we would need

$$
\#\left(\Lambda_{2}\right) / \mu\left(K_{m}\right)(c(x))^{N} \rightarrow n^{-N} a^{-N}
$$

uniformly for $K_{m}$ as $x \rightarrow \infty$. But

$$
0 \leq \frac{\#\left(\Lambda_{2}\right)}{\mu\left(K_{m}\right)}\left(\frac{a n}{c(x)}\right)^{N}-1 \leq \frac{S_{N}\left(r_{m+1}^{N-1}+r_{m}^{N-1}\right) \sqrt{N}(a n / c(x))}{V_{N}\left(r_{m+1}^{N}-r_{m}^{N}\right)} \leq \frac{N^{3 / 2}}{\Delta} \frac{a n}{c(x)} \rightarrow 0
$$

uniformly in $m$, where the coefficients $S_{N}, V_{N}$ satisfy $N V_{N}=S_{N}$. Therefore, we choose $\Delta$ small enough and then $n_{0}$ large enough that 


$$
\begin{aligned}
& \sum_{n=n_{0}}^{\infty} P\left(\max _{r_{n} \leq|p| \leq r_{n}+1} X(p) \geq \phi\left(r_{n}\right)\right) \\
& \leq \text { const } \sum_{n=n_{0}}^{\infty} \mu\left(K_{n}\right)\left(\phi\left(r_{n}\right)\right)^{-1}\left(\tilde{\sigma}^{-1}\left(1 / \phi\left(r_{n}\right)\right)\right)^{-N} \exp \left(-1 / 2 \phi^{2}\left(r_{n}\right)\right) \\
& \leq \text { const } \int_{r \geq n_{0} \Delta}(\phi(r))^{-1}\left(\tilde{\sigma}^{-1}(1 / \phi(r))\right)^{-N} \exp \left(-1 / 2 \phi^{2}(r)\right) r^{N-1} d r \\
& <\infty .
\end{aligned}
$$

So when we apply the Borel-Cantelli lemma, we obtain the conclusion of Theorem 3.1.

Theorem 3.2. Suppose $X$ is a continuous Gaussian random field with mean zero and variance one satisfying part (B) of condition (1.1); that is, there are positive constants $C_{1}, \delta_{2}, T_{2}$ such that

$$
\rho(p, q) \leq 1-C_{1}|p-q|^{\alpha} H(|p-q|)
$$

for all $p, q$ satisfying $0<|p-q|<\delta_{2}$ and $|p|,|q| \geq T_{2}$.

Suppose $X$ also satisfies condition (1.3); that is, there is a $\gamma>0$ sucb that

$$
\rho(p, p+q)=O\left(|q|^{-\gamma}\right) \text { uniformly in } p \text { as }|q| \rightarrow \infty \text {. }
$$

If, for any positive nondecreasing function $\phi(r)$, then $P\left(A_{\phi}\right)=0$.

$$
I(\phi)=\int^{\infty}\left(\tilde{\sigma}^{-1}(1 / \phi(r))\right)^{-N}(\phi(r))^{-1} \exp \left(-1 / 2 \phi^{2}(r)\right) r^{N-1} d r=\infty,
$$

Before giving the proof we need the following lemma.

Lemma 3.1. If Theorem 3.2 is true under the additional condition that, for large $r, 2 N \log r \leq \phi^{2}(r) \leq 3 N \log r$, then it is true without this restriction.

Proof. The proof of Lemma 3.1 is accomplished in the same way as for Lemma 3.1 in Qualls and Watanabe [4]. So we omit it.

Proof of Theorem 3.2. Let $r_{n}=2 n \Delta$, for large $\Delta$, and let

$$
D_{n}=\left\{p=\left(r, \phi_{1}, \cdots, \phi_{N-1}\right): r_{n} \leq r \leq r_{n}+\Delta, 0 \leq \phi_{i} \leq \pi, 0 \leq \phi_{N-1} \leq \epsilon\right\}
$$

for $\epsilon>0$. Let $G_{n}$ be the set of lattice points in $D_{n}$ obtained by dividing $N$-space into cubes with sides of length $1 / l_{n}$. Now we take $l_{n} \sim\left(\tilde{\sigma}^{-1}\left(1 / \phi\left(r_{n}+\Delta\right)\right)\right)^{-1}$.

Now by applying the Corollary to Lemma 2.3 , we can estimate the probabilities of the events $E_{n}=\left\{\max _{p \in G_{n}} X(p) \leq \phi\left(r_{n}+\Delta\right)\right\}$ for large $n$. The convergence of the left-hand side of (2.13) can be shown to be uniform with respect to all the sets $K_{n}$; for example following inequality (2.11), we would need $\#\left(\Lambda_{1}\right) / \mu\left(K_{n}\right)(c(x))^{N} \rightarrow$ $n^{-N} a^{-N}$ uniformly for $K_{m}$ as $n \rightarrow \infty$ which can be done as in the proof of Theorem 3.1. Then 


$$
P\left(E_{n}^{c}\right) \geq \text { const } \mu\left(D_{n}\right)\left(\tilde{\sigma}^{-1}\left(1 / \phi\left(r_{n}+\Delta\right)\right)\right)^{-N}\left(\phi\left(r_{n}+\Delta\right)\right)^{-1} \exp \left(-1 / 2 \phi^{2}\left(r_{n}+\Delta\right)\right)
$$

implies

$$
\sum_{n=n_{0}}^{\infty} P\left(E_{n}^{c}\right) \geq \text { const } I(\phi)=\infty .
$$

Next we show $P\left(E_{n}^{c}\right.$ i.o. $)=1$, which would imply the conclusion of Theorem 3.2. Since $\Pi_{k=m}^{\infty} P\left(E_{k}\right)=0$, we need to show

$$
1-P\left(E_{n}^{c} \text { i.o. }\right)=\lim _{m \rightarrow \infty}\left(P\left(\bigcap_{k=m}^{\infty} E_{k}\right)-\prod_{k=m}^{\infty} P\left(E_{k}\right)\right)=0 .
$$

Now, by Lemma 1.5 in Qualls and Watanabe [3], we estimate

by

$$
A_{m, n}=\left|P\left(\bigcap_{k=m}^{n} E_{k}\right)-\prod_{k=m}^{n} P\left(E_{k}\right)\right|
$$

$$
A_{m, n} \leq \sum_{m \leq i<j \leq n} \sum_{\mu=0}^{K_{i}} \sum_{\nu=0}^{K_{j}} \rho \int_{0}^{1} g\left(\phi\left(r_{i}+\Delta\right), \phi\left(r_{j}+\Delta\right), \lambda \rho\right) d \lambda,
$$

where $\rho=\rho\left(p_{i, \mu}, p_{j, \nu}\right), K_{n}=\#\left(G_{n}\right)=\#\left\{p_{n, \nu} ; \nu=1,2, \cdots, K_{n}\right\}$, and $g(x, y, \rho)=$ $(2 \pi)^{-1}\left(1-\rho^{2}\right)^{-1 / 2} \exp \left(-2^{-1}\left(1-\rho^{2}\right)^{-1}\left(x^{2}-2 \rho x y+y^{2}\right)\right)$.

Because $\left|p_{i, \mu}-p_{j, \nu}\right| \geq \Delta$ and because of condition (C), $\Delta$ can be chosen large enough that, for some positive constant $M$,

$$
\left|\rho\left(p_{i, \mu}, p_{j, \nu}\right)\right| \leq M((j-i) \Delta)^{-\gamma}, \text { for all } j>i \geq m,
$$

and $|\rho|<\gamma / 6 N$. For $m$ sufficiently large, Lemma 3.1 implies

$u^{2}((2 i+1) \Delta) \equiv 2 N \log ((2 i+1) \Delta) \leq \phi^{2}((2 i+1) \Delta) \leq 3 N \log ((2 i+1) \Delta) \equiv v^{2}((2 i+1) \Delta)$

for all $i \geq m$. Now

$$
\begin{aligned}
g(\phi( & (2 i+1) \Delta), \phi((2 j+1) \Delta), \lambda \rho) \\
& \leq(2 \pi)^{-1}\left(1-\rho^{2}\right)^{-1 / 2} \exp \left(-1 / 2\left(\phi^{2}((2 i+1) \Delta)-2|\rho| \phi^{2}((2 j+1) \Delta)+\phi^{2}((2 j+1) \Delta)\right)\right) \\
& \leq(2 \pi)^{-1}\left(1-(\gamma / 6 N)^{2}\right)^{-1 / 2} \exp \left(-1 / 2\left(u^{2}((2 i+1) \Delta)+(1-2|\rho|) u^{2}((2 j+1) \Delta)\right)\right) \\
& \leq(2 \pi)^{-1}\left(1-(\gamma / 6 N)^{2}\right)^{-1 / 2}[1 /(2 i+1) \Delta]^{N}[1 /(2 j+1) \Delta]^{N(1-3|\rho|) .}
\end{aligned}
$$

Since $l_{i} \sim\left(\tilde{\sigma}^{-1}(1 / \phi((2 i+1) \Delta))\right)^{-1}$, then

$$
K_{i} \sim l_{i}^{N} \mu(D) \sim \text { const }(2 i+1)^{N-1}\left(\tilde{\sigma}^{-1}(1 / \phi((2 i+1) \Delta))\right)^{-N} \quad \text { as } i \rightarrow \infty .
$$

From Lemma 3.1 and property (2.4), we see that $K_{i} \leq$ const $(2 i+1)^{N-1}(\log (2 i+1))^{N /(a-\epsilon)}$ for large $i$ and some $\epsilon>0$ such that $\alpha-\epsilon>0$. Consequently, we have, tor large $m$, 


$$
\begin{aligned}
& A_{m, \infty} \leq \text { const } \times \sum_{m \leq i<j<\infty} \frac{(2 i+1)^{N-1}(2 j+1)^{N-1}(\log (2 i+1))^{N /(a-\epsilon)(\log (2 j+1))^{N /(a-\epsilon)}}}{(j-1)^{\gamma}} \\
& \times[1 /(2 i+1)]^{N}[1 /(2 j+1)]^{N(1-3|\rho|)} \\
& \leq \text { const } \times \sum_{i=m}^{\infty} \sum_{k=1}^{\infty} \frac{(\log (2(k+i)+1))^{2 N /(a-\epsilon)}\left(\frac{1}{2 i+1}\right)\left(\frac{1}{2(k+i)+1}\right)^{1-3 N|\rho|}}{k^{\gamma}} \\
& \leq \text { const } \times \sum_{i=m}^{\infty} \sum_{k=1}^{\infty}\left(\frac{1}{k}\right)^{1-\delta+\gamma}\left(\frac{1}{2 i+1}\right)^{1+\delta-\gamma / 2}(\log (2(k+i)+1))^{2 N /(a-\epsilon) .}
\end{aligned}
$$

Since $\log ^{\beta}(x+y) \leq \log ^{\beta} 2 x+\log ^{\beta} 2 y$ for any $\beta>0$ and all $x, y \geq 1$, we see that this last sum is convergent if we choose $\delta$ so that $\gamma / 2<\delta<\gamma$. Therefore $\lim _{m \rightarrow \infty} A_{m, \infty}=0$, which completes the proof.

\section{REFERENCES}

1. W. Feller, An introduction to probability theory and its applications. Vol. II, 2nd ed., Wiley, New York, 1966. MR 42 \#5292.

2. J. Pickands III, Upcrossing probabilities for stationary Gaussian processes, Trans. Amer. Math. Soc. 145 (1969), 51-73. MR $40 \# 3606$.

3. C. Qualls and H. Watanabe, An asymptotic 0-1 behavior of Gaussian process, Ann. Math. Statist. 42 (1971), 2029-2035.

4. - Asymptotic properties of Gaussian processes, Ann. Math. Statist. 43 (1972), $580-596$.

5. D. Slepian, The one-sided barrier proolem for Gaussian noise, Bell System Tech. J. 41 (1962), 463-501. MR 24 \#A3017.

DEPARTMENT OF MATHEMATICS, UNIVERSITY OF NEW MEXICO, ALBUQUERQUE, NEW MEXICO 87106

DEPARTMENT OF APPLIED SCIENCE, KYUSHU UNIVERSITY, FUKUOKA, JAPAN 